\title{
Medizinisches aus Luzerner Sterbebüchern
}

\author{
Von Joseph Schürmann
}

Ähnlich wie die ab 1580 auf Weisung des Tridentinischen Konzils und auf Druck durch die weltliche Regierung nach und nach angelegten Taufbücher wurden mit der Zeit auch Totenbücher geführt, fast durchwegs in Latein. Aber es scheint dem Belieben der Pfarrer anheimgestellt gewesen zu sein, wie ausführlich sie ihre Angaben halten wollten. So finden wir vielfach nur Namen, und zwar nur solche von Erwachsenen, das heißt von Personen, die zur Kommunion zugelassen waren. Erst nach und nach treten Einzelheiten zu den Namen, und auch diese recht unterschiedlich. Dann bildet sich die Gewohnheit aus, zu vermerken, ob der Verstorbene noch die drei Sterbesakramente Beichte, Kommunion und Letzte Ölung hatte empfangen können. Wenn die sterbende Person bildungsunfähig - z.B. taubstumm gewesen und nie zur Beichte zugelassen war oder wenn der Tod so plötzlich eingetreten war, daß die Sterbesakramente überhaupt nicht hatten erteilt werden können, oder wenn der Sterbende die Hostie nicht mehr hatte empfangen können, dann wurde das mit der Zeit ins Totenbuch eingetragen, und bald muß sich das Bedürfnis gezeigt haben, niederzuschreiben, welches die besonderen Umstände gewesen waren, welche den Sakramentenempfang behindert oder gar verunmöglicht hatten. So sind auch Einzelheiten über Unfälle und andere Todesursachen festgehalten worden; einige davon vermögen heute noch unser Interesse zu wecken.

Wenn immer möglich sollte ein Katholik in geweihter Erde bestattet werden. Anderseits wollte man diesen Vorzug jenen nicht zugestehen, die der katholischen Konfession nie angehört oder sich durch eine letzte verwerfliche Handlung der himmlischen Gnaden für unwürdig erwiesen hatten. Darum bildete sich in jenen Gemeinden, welche an der Grenze zwischen katholisch und reformiert lagen, die Gewohnheit heraus, über die Grenze hinweg die Leichen auszutauschen. 1754 ist im Totenbuch Reiden ausdrücklich von einem «Gegenrecht» gegenüber Zofingen die Rede. - Unter zahlreichen einschlägigen Fällen sticht besonders der einer Dame aus vornehmen französischen Kreisen hervor, die 1780 Patientin von Micheli Schüpbach gewesen sein muß, jedenfalls von Langnau her auf dem Friedhof von Marbach ihre letzte Ruhe fand. 
Eine andere Eigentümlichkeit alter Zeiten bestand darin, daß die Leute jener Pfarrei irgendwie verbunden blieben, in welcher sie getauft worden waren. Das führte dazu, daß man ihrer in der heimatlichen Pfarrei nach dem Tod mit einer Trauermesse, «exequiae» genannt, gedachte, selbst solcher Leute, die sonst keinen Kontakt mit der Heimat mehr gehabt hatten. Wir wissen heute noch, wer auf einer Pilgerfahrt oder in einer Thermalkur gestorben ist, so in Baden oder Leuk oder in einem der heute nur noch dem Namen nach bekannten Bäder. Viel zahlreicher sind die Namen von Ausgewanderten und von Söldnern, über die auf irgendeinem Weg manchmal um Jahre verspätet - noch eine Nachricht in die alte Heimat gelangt ist. Die folgenden Beispiele handeln zwar von Exsöldnern, aber sie zeigen ganz besonders düstere Schattenseiten des Söldnertums. «... qui coactus crudeli Regi brandenburgensi ex Prussia 11 annos in bello serviit, hic in lecto obiit» (Luthern 1760) gemahnt an das Schicksal Ueli Bräkers, des «Armen Mannes aus dem Tockenburg». ${ }^{1}$ Noch dramatischer ist das Beispiel aus Entlebuch (1803) «... a Galliae ducibus ante 40 annos ob levem alapam ad insolubiles triremes damnatus tempore revolutionis gallicae liberatus, laboriosissimus vivens... » schwere Sühne für eine Ohrfeige im Verlauf einer Insubordination.

Einzelheiten über die verstorbenen Personen sind - wie bereits angetönt sehr unterschiedlich vermerkt. Der Unterschied konnte sogar in der Person des Pfarrers selber liegen, ob er sich für besondere Umstände überhaupt interessierte und für welche aus einem recht breiten Spektrum. Sobald man die Aufzeichnungen der verschiedenen Pfarrherren zu verschiedenen Zeiten miteinander vergleicht, lassen sich allerlei Varianten feststellen. Da hatte der eine das Bedürfnis, außergewöhnliche Todesfälle als «Strafe Gottes» hinzustellen, so etwa in Schüpfheim 1762: «die festivo neglecta missae auditione cum servo servilia peragens sine licentia, a lapide de rupe cadente tactus immediate obiit» oder in Root 1777 von einem 64jährigen Mann: «filios inhumaniter verberans ipse tandem reculpavit poena talionis ictu arboris capite suo impacto». - Da und dort kommt in Notizen zu einzelnen Todesfällen ein besonderes Interesse der Geistlichen an Medizin und Anatomie zum Ausdruck. Wenn 1698 in Richenthal zu einem Hufschlag mit letalem Ausgang bemerkt wird, «dura atque pia matre offensus», so stellt das allerdings eine große Ausnahme dar. Den Namen von Leuten, die von einem Baum gestürzt sind, wird gelegentlich beigefügt «fracta cervice», und einmal wird bei einem solchen Fall Chorda dorsalis sogar ins Deutsche übersetzt, «die Rückenschnur» sei gebrochen gewesen. - Zweifellos im Zug 
der Aufklärung finden sich ab 1770 immer mehr Bemerkungen zu den einzelnen Todesfällen über Umstände, in denen die Leute gelebt hatten, und in Zusammenhang damit auch über letzte Krankheiten und vermutete Todesursachen. Am konsequentesten haben das zwei Pfarrer Häfliger in Hochdorf, Onkel und Neffe, zwischen 1777 und 1830 getan. In einer Rubrik «modus obitus et locus sepulturae» vermerkten sie eine Art Kurzdiagnose. Darüber wird später noch mehr zu sagen sein.

Das Alter ist vielfach nur dann besonders angegeben, wenn seine Höhe auffiel, am einen Ort schon jenseits von 60 , anderwärts, wenn es 80 und mehr betrug. Diese Angaben sind allerdings vielfach nur recht ungefähr, bei weitem nicht etwa anhand des Taufbuchs kontrolliert und genau nachgerechnet. Wir schließen das daraus, daß die Zahl der angeblich 100 - und noch mehr jährigen - 109 und 117 kommen vor - mit der Zeit deutlich zurückgeht. (Zu diesem Kapitel ein Beispiel, das zwar nicht Kirchenbüchern entnommen ist, jedoch ein Gebiet betrifft, auf dem wir noch größere Genauigkeit voraussetzen würden als bloß in zivilen Quellen: 1763 stirbt in der Garde zu Wien der angeblich 112jährige Profos Oswald Zülli aus Sursee, seit $1698 \mathrm{im}$ Dienst der Garde. Das Taufbuch Sursee führt drei Gleichnamige auf, aber auch der älteste von ihnen ist erst 1664 geboren, der jüngste 1669.) Vermerkt werden konnte auch eine vom Üblichen abweichende Zahl Ehen, die der Verstorbene hinter sich gebracht hatte - einmal sind deren sieben verzeichnet - oder eine große Zahl von Kindern: 17 Kinder in 24 Jahren hatte eine Frau seinerzeit geboren, oder 159 Nachkommen in drei Generationen sind 1826 in Triengen beim Tod einer 90jährigen Witwe verzeichnet. Berufe werden nur selten erwähnt, am häufigsten aber doch die Hebamme, hie und da noch mit einem lobenden Zusatz.

\section{Einzelne Todesursachen}

\section{Epidemien}

In die Zeit, in welcher die ersten Sterbebücher angelegt worden sind, fallen die zwei letzten Pestepidemien, welche das Luzernbiet heimgesucht haben. Vom Seuchenzug von 1594 finden sich keine Spuren, zahlreiche dann aber vom letzten 1628/29. Da und dort wird bloß bemerkt, die Pest habe gewütet, ohne daß genau angegeben wird, wer daran gestorben ist; anderwärts, so in Schüpfheim, ist die Reihe der Pesttoten seitenlang. - Letzte Andeutungen über den Aussatz finden sich in den Städten Sursee und Wilisau, weil eben zu 
einer Stadt gehörte, daß sie auch ein «Siechenhaus» führte. In Sursee finden sich 1614 bis 1627 die Zusätze «leprosus» und «Undersiech» ... was möglicherweise ein Mißverständnis für «Sundersiech» darstellt; zwei Willisauer Fälle gehören in die Jahre 1602 und 1610. (In der Stadt Luzern, wo wahrscheinlich mehr solche Fälle vorgekommen sind, setzt das Totenregister erst 1700 ein.) Wenn 1742 von einem Mann aus Neudorf vermerkt wird, er sei «Lucernae in domo leprosorum» gestorben, darf das wohl nur noch mit «im Spital zu Luzern» gedeutet werden. - Ergotismus, St.-Antonius-Feuer, war schon in den letzten Jahren des 17.Jh. im Luzernbiet aufgetreten, eingehender beschrieben werden kleine Epidemien erst 1709 und $1713^{2}$.

Ob ein Fall wie der folgende der genannten Ursache zuzuschreiben wäre, muß dahingestellt bleiben, da die Vorgeschichte nicht bekannt ist. In Schongau wird 1697 aufgezeichnet, eine Frau sei in Luzern, und zwar in Gegenwart des Dr. Cysat und des Chirurgen Schobinger gestorben; ihr hatte man einen Arm amputiert. Obschon aus den Endemien von 1709 und 1713 über Todesfälle in der Gegend von Willisau berichtet wird, erscheinen in den Sterbebüchern keine brauchbaren Hinweise. - Für Starrkrampf und Milzbrand läßt sich 1826 aus Hochdorf je ein Fall anführen: «Opisthotono seu tenesmo, spasmate in collo» bei einem 38j. Mann, «Anthrace» bei einem $65 \mathrm{j}$. Bauern. - Eine Tollwutendemie muß es um 1670 in Ruswil gegeben haben: Vier Knaben sind «morsu canis rabidi» gestorben. Später finden wir noch ganz vereinzelte Fälle in benachbarten Gemeinden verzeichnet. -«Influenza» wird als Epidemie 1747 erstmals in der Literatur beschrieben. Es muß auch bei uns längst vor der ersten besser bekannten Epidemie von 1890/91 solche gegeben haben, wie da und dort Häufungen von «febris calida», «febris catarrhosa» auch bei jüngeren Leuten dartun. - Vereinzelt kommt «Dysenterie» immer wieder vor. Eine eigentliche Epidemie von «Roter Ruhr» hat 1770/71 das Land heimgesucht. - 1813/14 zogen alliierte Truppen durchs Schweizer Mittelland Richtung Burgund und Frankreich. Sie hinterließen eine Fleckfieberepidemie. Im Kloster St. Urban muß ein Lazarett bestanden haben. Dort starben zwei Männer aus der weiteren Umgebung, die offensichtlich zur Krankenpflege aufgeboten worden waren: Aus Luthern der ledige J. F. «morbo contagioso», aus Winikon der 54j. N. L., von dem es heißt, er habe in St. Urban kranke deutsche Soldaten gepflegt. Pocken sind um 1800 mehrfach erwähnt, am ausführlichsten in Großdietwil, wo der Pfarrer seinen Kummer darüber ins Totenbuch schreibt, daß die Eltern sich der Impfung gegenüber ablehnend verhalten, und daß es ihnen gleichgültig sei, ob ihre Kinder stürben oder am Leben blieben. - Diphtherie 
und Scharlach sind nicht auszumachen, auch Typhus nicht, obschon es immer Bazillenausscheider gegeben haben muß, die von Zeit zu Zeit aus einem Dorfbrunnen eine Ansteckungsquelle machten. - Dem Wort «hectica» als Verdacht auf Tuberkulose begegnen wir in Schongau schon 1701 ... ganz vereinzelt, häufiger dann in den bereits erwähnten Aufzeichnungen aus Hochdorf, hier nun gepaart mit «Phthise» und mit «febris», auch «tussis pituitaria». Ein «hecticus» aus Sempach stirbt 1789 auswärts, wo er in einer Molkenkur Heilung gesucht hatte. Nicht gar leicht zu deuten ist der öfters vorkommende Terminus «haemorrhagia», solange er allein steht. Ist noch «suffocatus» beigefügt, so muß es sich wohl in den meisten Fällen um Blutungen aus einer tuberkulösen Caverne gehandelt haben. Das Wort spielt auch in anderem Zusammenhang eine Rolle: In Luthern stirbt 1765 eine Virgo, über welche festgehalten wird «in monasterio ob sanguinis eructationem manere nequivit».

\section{Innere Krankheiten}

Wenn wir bedenken, daß lange Zeit zum Erkennen einer Krankheit mehr auf das abgestellt wurde, was der Patient als Beschwerden vorbrachte ... oder gar auf das, was die Person zu erzählen wußte, welche «für einen Kranken zum Arzt ging» (und dessen Urin mitbringen mußte) ... als auf eigene Untersuchung mit dem klopfenden oder tastenden Finger und dem lauschenden Ohr des Arztes, begreifen wir auch einen Wirrwarr von Bezeichnungen und eine Unschärfe der Begriffe, namentlich noch dann, wenn solches zuletzt von einem Nichtfachmann ins Totenbuch gesetzt wurde. Alle unsere Fälle gehören noch der Zeit an, die weder Pulszählen noch gar Fiebermessen kannte. Ältere und alte Leute starben weitherum «senio confectus». Zwei der genannten unklaren Begriffe waren «febris» und «phthisis». Wohl übersetzen wir heute «febris» gemeinhin mit «Fieber» und unterstellen dabei, daß die Körpertemperatur gemessen und zu hoch befunden wurde. Aber es bleibt zu bedenken, daß es vor 1800 gar keine objektive Bestimmung der Körpertemperatur gegeben hat. Wohl hatte das Wort seine Berechtigung - im heutigen Sinn - wenn die Stirn des Kranken mit der Hand heiß anzufühlen war und wenn gar dazu der Puls auffallend rasch schlug. Aber schon die Tatsache, daß es neben einer großen Zahl anderer charakterisierender Adjektive auch noch ein «kaltes» Fieber gab, läßt vermuten, daß beim Volk und bei den Ärzten - auf dem Land bei den Scherern und Badern, den «Chirurgen» - und beim Pfarrer «febris» ganz ein- 
fach «Krankheit» bedeutete. Als andere Adjektive zu «febris» seien erwähnt «gastrica, biliosa» (letzteres vermutlich bei Gelbsucht ... das Wort «icterus» wird nie gebraucht) «calida»; «putrida», wenn es zu Eiterbildung gekommen war, damals mit «Faulfieber» übersetzt; «pituitaria» sollte besonders viel und vielleicht auch besonders gearteten Auswurf charakterisieren. - «Phthisis», wörtlich "Verderbnis», erscheint bei Verstorbenen aller Altersklassen jenseits der Kindheit. Hingegen lesen wir nie von Kachexie. Daraus darf der Schluß gezogen werden, "phthisis» sei für das Endstadium sowohl von Tuberkulose wie von Krebsfällen verwendet worden. 1791 wird in Root «Auszehrung» einer 70j. Frau anschaulicher mit «super ossa pellis» umschrieben. - Auch unter «Krebs» verstanden unsere Vorfahren noch nicht das gleiche wie wir. Sie wußten nichts von Zellen und konnten diesen auch kein bösartiges Wachstum zuschreiben. Wie noch vor kurzem im Volk wurde auch bei Gebildeten sogar zwischen «Geschwulst» und «Geschwür» nicht scharf unterschieden; so auch in Neudorf 1784 bei einem Exsöldner, «pede laesus malo cancrino obiit», was daran denken läßt, es könnte sich um einen der früher häufigen Fälle von «offenen Beinen», Ulcera cruris postthrombotica, gehandelt haben, auf keinerlei Behandlung ansprechend und darum als «krebsig» erachtet. «Ex insanabili ulcere», wie schon 1643 bei einer Frau in Inwil festgehalten ist, «dolore ex vulneribus cancrosis ortis per plures annos» (Sempach 1783) «foedo ulcere in crure» (bei 33j. Frau 1826 in Hochdorf) sind andere Beispiele zu diesem Kapitel. Mammakarzinom ist nur einmal unverkennbar erwähnt, 1768 in Neudorf als «cancro in uberibus». Auch «diuturna uberum aegritudine pressa» (Beromünster 1690) dürfte gleich zu deuten sein. Ähnlich leicht richtig zu beurteilen war wohl auch Hautkrebs im Gesicht, als «malo cancrino in facie» festgehalten (Neudorf 1803) oder «scorpione vultum naresque deposcente lente contabuit». Auf Malignom im Verdauungstrakt dürfen wir schließen, wenn wir lesen, "plurium annorum spatio stomachicus et cibum paucissimum aegre deglutiens» (Neudorf 1781). «Tumor nodosus in collo» (Geiss 1765) muß der früher häufige Kropf gewesen sein. - «Hydrops» war wenigstens ein Krankheitszeichen, das sich schon bei der Inspektion oder gar in der Schilderung durch Drittpersonen klar zu erkennen gab, auch wenn niemand die verschiedenen Ursachen dieser Wassersucht auseinanderhalten konnte. Schon 1785 wird in Root von einer $44 \mathrm{j}$. Frau geschrieben, sie sei mehrmals am Bauch punktiert worden. Die Häufigkeit, mit der «Hydrops» als Todesursache aufgezeichnet wird, läßt die Kausalkette Polyarthritis - Endocarditis - Vitium cordis - Insufficientia cordis vermuten. 


\section{Altersleiden}

sind sehr häufig nur mit «senio», «senio confectus» aufgezeichnet worden. Aus Root hingegen vernehmen wir 1779 «ad statum quasi infantis reductus» und 1787 von einem 80j. ehemaligen «Leutnant» (er war seinerzeit lokaler Vertrauensmann der Regierung in militärischen Angelegenheiten gewesen) «per annos in imbecillitate perpessus».

\section{Apoplexie}

Immer aus dem gleichen Grund - Ableben ohne Empfang der Sterbesakramente - sind die Umstände in vielen Fällen im Sterbebuch festgehalten worden, wenn die Person tot aufgefunden wurde, sei es am Morgen im Bett Bettler im Stall - sei es auf der Straße oder auf dem Felde, wo der Tod während der Arbeit eingetreten war. Ganz besonders beeindruckt waren die geistlichen Herren, wenn das brutale Ereignis einen Kirchgänger getroffen hatte oder gar den Organisten oder den Sigristen während des Gottesdienstes. Auf den Sitz des letal wirkenden Gefäßverschlusses können wir in den allerwenigsten Fällen sicher schließen. Im Volksmund werden ja Apoplexia cerebri und Coronarverschluß heute noch mit «Schlag» gleichgesetzt. Interessant für uns kann jener Fall eines $67 \mathrm{j}$. Mannes sein, von dem der Pfarrer von Root 1787 schreibt, er habe zunächst eine «apoplexia» gut überstanden: «apoplexia tactus et postea liberae menti restitutus». Von einer 66j. Witwe wird 1766 in Neudorf berichtet: «Frigidus apoplexiae fluxus illam ex una parte faciei et corporis delumbem fecit.» Das läßt sich wörtlich mit dem früher geläufigen Wort «Schlagfluß» übersetzen.

\section{Geisteskrankheiten}

Ausdrücke wie «obsessa», «melancholia», «continua phrenesis», «corruptio mentis», «fatuus», «ratione carens», «correpti ingenii» können wir ins moderne Schema nicht mehr richtig einreihen. Sie wurden ja auch wahllos durcheinander gebraucht, da es eine Differentialdiagnose noch gar nicht gab.

\section{Nervenkrankheiten}

Wenn wir über eine 22j. Frau aus Sempach 1787 lesen «dudum histerico morbo dire vexata extincto vix non per annum oculorum lumine», so gemahnt uns das an die noch nicht weit zurückliegenden Zeiten, in denen erst die Opticusatrophie endlich zur Diagnose «Multiple Sklerose» führte. - 
«Gravissime omnibus membris tremebundus» ist schon 1668 in Großwangen notiert worden und zeigt, daß die Parkinsonsche Krankheit zu allen Zeiten vorgekommen ist.

\section{Epilepsie}

Zahlreich sind die Fälle, bei denen «morbo caduco» zum Namen des Verstorbenen gesetzt wurde, wohl allemal dann, wenn der epileptische Anfall mit Tod ausgegangen war. Ab und zu wird angedeutet, zum Zwischenfall sei es unter besonderen Umständen gekommen, so etwa wenn der Patient ertrunken war.

\section{Hernien}

Die ehemals geläufige Bezeichnung war «ruptura», mehr Riß als Bruch, von «Hernia» ist nirgends die Rede. 1664 stirbt in Pfeffikon ein Mann, «dum rupturâ curandum se alicui extraneo et vagabundo tradidisset», der also von einem fahrenden Bruch- und Steinschneider operiert worden war. (Bei einem einheimischen Wundarzt-Bruchschneider finden wir im Totenbuch die Berufsbezeichnung «operator».) Das ist der erste einer ganzen Reihe von Fällen, denen immer wieder eigentümlich ist, daß sich die Hernienträger nicht beim nächstwohnenden «Chirurgen» hatten operieren lassen, vielmehr in weiterer Entfernung Hilfe gesucht hatten. (Eschle ${ }^{3}$ berichtet, ein in Escholzmatt tätiger «operator» sei aus dem Bernbiet weggewiesen worden, weil er Hernien «per modum castrationis» behandelt habe ... dies immerhin interessant als Hinweis auf eine der damals üblichen Operationsmethoden. «Weggewiesen» könnte auch so gemeint sein: Der Operator ließ sich ins nahe Bernbiet rufen und handelte dort wie ein fahrender Bruchschneider. Die "Wegweisung» könnte Konkurrenzneid entsprungen sein.) Wahrscheinlich - eine Diagnose fehlt allerdings im Sterbebuch - der berühmteste Fall, der analogerweiser hieher gerechnet werden darf, ist der des Pfarrers Joseph Xaver Schnyder von Wartensee in Schüpfheim: Erst 34jährig hatte er 1784 bei einem berühmten Chirurgen in Straßburg Hilfe gesucht und ist nicht mehr zurückgekehrt.

\section{Appendicitis}

Eingangs haben wir darauf hingewiesen, daß es Fälle gab, in denen die sterbende Person wohl noch «bei Verstand» gewesen sein muß - denn ihr 
konnte noch die Beichte abgenommen werden - jedoch der Geistliche davon absehen mußte, noch die Hostie zu reichen. So stirbt in Pfaffnau 1777 ein 12j. Mädchen «cum absolutione et unctione extrema munita, dum ob qualitatem morbi S. hostiam deglutire nequivit». Oder 1683 in Beromünster ein 14j. Knabe «ob stomachi nauseam synaxin sumere non potuit» noch deutlicher in Eich «ob reverentiam SS. Sacramenti tantum confessa et S. oleo munita» und in Knutwil 1763 «ob vomitum non accepta Eucharistia». Daß man unter solchen Umständen in Betracht ziehen darf, diese Leute seien an der Folge einer Appendixperforation gestorben, mag erst recht aus dem ausführlichen Bericht hervorgehen, den wir 1765 im Totenbuch Ufhusen finden: Ein Kapuziner befand sich auf dem Weg von Luthern zurück in sein Kloster in Sursee. Mit ersten Beschwerden kehrte er am 29. Juni im Pfarrhof Ufhusen ein, «stomachi doloribus vexatus, quibus saepius cruciabatur; morbi vis ad sequentem diem nil remittebat, sed insuper omnibus remediis a D. medico Mengis adhibitis, morbi status decima tandem die ad extrema pervenit, obstructiones alvi, continui vomitus cum excrementis, icterus, colica biliosa ...» also der fast lehrbuchmäßige Ablauf eines Ileus. In allen diesen Beschreibungen vermissen wir nur das damals in der medizinischen Literatur weitverbreitete Wort «Miserere» für den Tod an Darmlähmung. War es hierzulande nicht üblich, weil ein Gebet um göttliches Erbarmen mit diesem Wort beginnt?

\section{Würmer}

Eingeweidewürmer sind als Todesursache erwähnt 1788 in Sempach bei einem 7jährigen Knaben als «vermium abundantia suffocatus» und in Hohenrain 1807 und 1809 bei Knaben mit «dolore vermium per plures dies cruciatus».

\section{Prostatahypertrophie}

Wird sie nur ganz am Rande gelegentlich mehr angedeutet als erwähnt, weil es etwa als unschicklich galt, von Krankheiten der Harn- und Geschlechtsorgane zu sprechen? Oder weil lange Zeit die von Prostatahypertrophie herrührenden Beschwerden mit «Steinleiden» gleichgesetzt wurden, und die Kranken bei den herumziehenden Steinschneidern Hilfe gesucht hatten? Und ob dem einen oder andern nicht das Leben noch verlängert worden ist, weil nach dem «Steinschnitt» eine Urinfistel entstanden war? Aber selbst Tod an «Steinleiden» findet sich nur selten aufgezeichnet, so 1696 in Ufhusen 
als «laborans calchalo», ähnlich in Sempach 1789 von einem 76j. Mann und in Willisau 1773 bei einem 66j. Mann «calculi morbo dirissimo», in Eschenbach 1773 von einem erst seit zwei Jahren verheirateten Mann «lapidibus nephriticis subito obtusus»... zur Zeit, als dort bereits ein «operator» wirkte. Um so überraschender dann die Häufung von Diagnosen nach 1800 in und um Römerswil; in Sempach 1827 ein 70j. Mann «ischuria», in Geiß 1834 ein $77 \mathrm{j}$. «stranguria diu vexatus», in Römerswil:

$181576 \mathrm{j}$. «passione dysurica per aliquot menses decumbens»

$181565 \mathrm{j}$. «stranguria laborans et putrida febre consumptus»

$182365 \mathrm{j}$. «per totum annum decumbens ischuria vel humore putrido in vesica laborans».

Und das alles hatte ein Pfarrer aufgezeichnet, von dem 1833, nachdem er 67 j. gestorben ist, sein Nachfolger schreiben wird, er habe an «retentio et stagnatio urinae» gelitten.

\section{Genußmittel}

In den Ratsprotokollen stoßen wir immer wieder auf Fälle, in denen von einer leichtsinnigen oder fahrlässigen Handlung die Rede ist, begangen in einer «Wynfüechti». Das Wort zeigt uns die hauptsächlichste Art des Alkoholmißbrauchs; gebrannte Wasser treten erst später in Erscheinung. Auf dem Land wurde anstelle von Wein vergorener Obstsaft genossen, und die Trester davon wurden gebrannt. Indirekte Folgen des Alkoholmißbrauchs zeigt uns 1690 der Zusatz zum Namen einer verstorbenen Frau in Richenthal: «Ad paupertatum reducta, cuius bona maritus deglutivit.» Als Todesursache werden aber Alkoholismus und seine Folgen nur selten und in späterer Zeit erwähnt, wieder abhängig von der persönlichen Einstellung des Pfarrers zum Problem. In Schongau wird 1735 von einem Ortsfremden festgehalten «aqua adusta nimis impletus per 50 horas exhalans et jacens discessit, o Vae!» ... in Root 1780 von einem 56j. Zimmermann «bibendo abundantias aquas adustas et phlebotomia nimiam sanguinis copiam profundendo paucos inter dies obiit», und 1782 von einem 62j. Junggesellen «finem vitae attigit quam piscando vino et musto adusto transegit», 1791 von einem 58j. ledigen Knecht «musto et aquae vivae aliquantulum dedictus», 1787 von einem 68j. Mann fast beschönigend «aliquantulum bibulus». - Der Pfarrer von Root, dem wir diese Einzelheiten verdanken, weiß aber auch 1791 von einer 36j. Frau zu melden, sei sie eine «singularis amatrix Tabaci fumigandi» gewesen ... immerhin 100 Jahre nachdem die 
Regierung das Verbot des «Tabacktrinckhens» hatte aufheben müssen, weil es einfach nicht durchzusetzen war.

\section{Frauenkrankheiten und Geburtshilfe}

Spärlich sind Hinweise auf gynäkologische Leiden als Todesursache. Zum einen «sprach man nicht von solchen Dingen», zum andern mögen die Geistlichen lieber davon abgesehen haben, darüber etwas zuhanden der Nachwelt festzuhalten. Aus Römerswil wird 1807 von einem 15j. Mädchen behauptet, es sei «suffocata in eruptione primorum menstruorum», 1819 aus Sempach bei einer 19jährigen; «retentis menstruis» und «ex obstructione menstruorum» 1784 bei einer 27jährigen in Neudorf, «obstruentibus menstruationibus suffocavit» 1809 bei einer $40 \mathrm{j}$. Frau in Ufhusen. - Demgegenüber lesen wir 1788 in Neudorf von einer 38j. Frau: «Haemorrhagia obiit ... mortem praematuravit terror ignis ante fenestras ab incendio parati», 1801 in Neuenkirch von einer Frau «sanguinis fluxu per hiemem decumbens»; da drängt sich Verdacht auf Myom auf. 1792 ist in Neudorf eine Frau zwei Wochen nach einer Fehlgeburt gestorben, in Sursee sind 1799 gleich zwei Fälle von «abortus in nimium timorem incendii» erwähnt. - Von Abtreibung ist nur einmal die Rede, 1817 in Altishofen bei einer 17jährigen.

Die ganze Grausamkeit alter Geburtshilfe, auf dem Land im Notfall von «Chirurgen» geleistet, geht aus Schilderungen wie diesen hervor: «Vagabunda partui proxima, cuius infantem mortuum ex utero materno rescidit nostri pagi chirurgus M.L. Hunc infantem, elemosyniis acceptis, cum duobus aliis Schattdorfium transmisi quo intercedente Dei genetrice, post varia data signa vitae a parocho eiusdem loci baptizatus est ibique sepultus.» Es soll also an dem für tot gehaltenen Kind noch ein Wunder geschehen sein (Oberkirch 1751). - Von einem beerdigten Kind: «In utero materno baptizatus et post mortem manu chirurgica concissus» (Pfeffikon 1765). «Inter periculosissimum et gravissimum puerperium una cum prole inter manus duorum chirurgorum et obstetricis obiit» (Römerswil 1823, 36j. Frau). - Oberstes Ziel der Geburtshilfe war damals, den Vorgang so weit zu lenken, daß das Kind, wenn immer möglich, noch getauft werden konnte. 1636 ist in Escholzmatt aus einem Visitationsbericht ins Taufbuch abgeschrieben worden: «3) Obstetrices a parochis diligenter supra suam functionem examinentur ac instruentur et tandem speciali iurato ad fidelem sui muneris executionem obstringantur.» Darum finden sich zu den Namen beerdigter Kinder Formulierungen wie diese: 
«in utero baptizatus» (vielfach)

«ad caput usque natus a chirurgo baptizatus» (Pfeffikon 1783)

«in porrecta manu ab obstetrice baptizata, postea in utero defuncta» (Pfeffikon 1772)

«In puerperio ... baptizatus in porrecta manu» (das Kind) «infante ac illo in utero iam defuncto et ipsa obiit», wenn die Mutter den Eingriff nicht überlebte. - «Ex modo matris extincto corpore mediante sectione extractus et sub conditione baptizatus» (Triengen 1775). - «Iuxta virum dormientem uxor circiter 8 per menses praegnans, aetate 42 annorum ... infans mortuus a chirurgo ex utero materno secatus est» ist eine weitere Schilderung eines solchen Dramas aus Schwarzenbach 1831. Ähnlich 1775 aus Ufhusen: ... (vom sonntäglichen Kirchgang) «S. synaxi refecta domum rediens in via improvise obiit adhuc infantem in utero habens, aperta matre infans quasi mortuus inventus et tamen baptizatus est.»

Auf Placenta praevia können wir heute noch schließen, wenn wir lesen: «partui proxima sanguinis fluxu subito obiit» (Hohenrain 1781) oder: «per nimium praematurum puerperium et sanguinis fluxu debilitata» (Römerswil $1812,37 \mathrm{j}$.$) .$

Auch die Diagnose Eklampsie läßt sich noch rekonstruieren: «partu laborans, deliquio extincta» (1798 Pfeffikon), «in puerperio loquela et fore etiam sensibus destituta» (Root $179037 \mathbf{j}$ ), «quae pridie in puerperio animi deliquium passa, subito extincta» (Hohenrain 1761). «Hydropisi durante puerperio correpta» (Pfaffnau 1693). «Puerpera, rationis non compos» (Root 1726) und ganz besonders deutlich: «Ultra IV menses a diversis medicis male tractata, jurantibus eam laborare hydropisi, tandem sensu et viribus corporis destituta non sine miraculo $\mathrm{S}$. Crescentiae ... sine labore peperit masculum ... altero die obiit» (Neudorf 1776) oder «hydrope obiit, sectus ex mortuae matris utero filius vivens» (Entlebuch 1770; der Knabe starb nach drei Monaten). Verblutung nach der Geburt wird ebenfalls beschrieben: «Effusione sanguinis post partum» (Sempach 1828 24j.). «Relictis secundinis» läßt offen, ob die Frau verblutet oder an Kindbettfieber gestorben ist. - «Statim cum peperit, ex profluvio sanguinis occumbens» (Pfaffnau 1721) läßt an Wehenschwäche in der Nachgeburtsperiode denken. Ebenso, wenn auf die Notiz vom Tode des einen neugeborenen Zwillings folgt: «Hunc suum infantem pia mater secuta est fluxu sanguinis miserrime laborans» (Pfeffikon 1801). - Kindbettfieber war so häufig, daß irgendeine Bezeichnung dafür gar nicht ins Totenbuch gelangte. Hierher rechnen 
müssen wir alle die vielen Frauen, von denen es nur heißt, sie seien als «puerpera» oder «in puerperio» gestorben. Aufgefallen sind besondere Zeichen zweimal aber doch: «Contraxit febrim et mortem ex partu infeliciter saccadente» (Sempach 1776) und "contracta ex puerperio matricale putredine» (Sempach 1780). - Ab und zu wird dem Namen einer verstorbenen Frau noch beigefügt, sie habe schwerste Geburten durchgemacht; kein Kind habe den Vorgang überlebt. Sogar beim Namen eines verstorbenen Mannes kann sich ein solcher Hinweis finden: «Iam quinquies ejus uxor E.S. tali modo infauste peperit, ut nullus infantium solemnem susceperit baptismum» (Pfeffikon 1781).

\section{Kindersterblichkeit}

Wie enorm sie war, geht sehr deutlich aus den sorgfältigen Aufzeichnungen in den Sterbebüchern von Hochdorf, 1777-1830, hervor: Ein Viertel aller Todesfälle betreffen Kinder unter 5 Jahren; auch die sub partu gestorbenen sind dabei mitgezählt. So auffällig auch damals Ernährungsstörungen gewiß schon waren, wir vermissen jeglichen Hinweis darauf. Wenn überhaupt - so gerade im genannten Hochdorf - eine Todesursache aufgezeichnet wurde, dann war es «convulsionibus» oder «convulsionibus suffocatus».

\section{Selbstmord}

Jahrhunderte alte Praxis der Kirche war, Selbstmördern die Ruhe in geweihter Erde zu versagen. Wenigstens da, wo die Umstände nicht anders als mit Suizid gedeutet werden konnten, wurde der Leib des Verworfenen vom Scharfrichter in der Nähe des Galgens verscharrt. Um die Mitte des 18. Jahrhunderts begann die Aufklärung auch in dieser Hinsicht ihre Früchte zu tragen. Die Regierung ließ sich über solche Fälle Bericht erstatten und befahl den Pfarrern, Selbstmörder in die Friedhöfe aufzunehmen. Sie pflegten die quasi erzwungenen rituellen Handlungen nach Sonnenuntergang auszuüben und untersagten dem Sigrist, dazu das Totenglöcklein zu läuten. «Sine luce, sine cruce» finden wir im Sterbebuch eingetragen. Stilles Auflehnen gegen die obrigkeitliche Verfügung schimmert durch bei "gratiatus a superioritate civili» (Schüpfheim 1774), und das Vorgehen insgesamt wird dargestellt mit «aliquoties melancholia gravi oppressus ex mandato Senatus sine exequiis et sonitu campanarum ad crepusculum sepultus» (Root 1778, 50j. lediger Mann). Aber auch ein gewisses Interesse an Einzelheiten eines solchen Falles ist uns erhalten geblieben: Ein 55j. 
Mann hatte ein beträchtliches Erbe vertan, zuletzt mit Alkoholmißbrauch; er endete «quasi a perito carnifice laqueo suspensus» (Flühli 1805).

\section{Unfälle}

Obenan steht die Zahl der Ertrunkenen. Die näheren Umstände waren so ziemlich dieselben wie heute; sie reichen vom Bootsunglück über Mitgerissenwerden bei Hochwasser bis zum verkappten Selbstmord. In der zweiten Hälfte des 18. Jahrhunderts kam die Stallhaltung des Rindviehs auf, und vor die Ställe wurden Jauchegruben gebaut. Damit entstand auch schon eine Falle für kleine Kinder, und wenn eines auf diese Weise seinen Tod fand, wurde das im Totenbuch vermerkt. Ein Pfarrer macht sogar den Eltern Vorwürfe, sie würden ihre Kleinen zuwenig beaufsichtigen. - Mehrmals sind größere Brandunglücke erwähnt; die auf dem Land durchwegs aus Holz gebauten Häuser entzündeten sich um so leichter, als auch für die Beleuchtung nur offene Flammen verwendet wurden. Daß wenigstens das Wesen der CO-Vergiftung, wenn auch noch nicht die chemische Konstitution des giftigen Gases bekannt war, geht aus einer Bemerkung hervor, die 1775 in Marbach festgehalten wurde: «Vapore ex ignitis carbonibus calefacti stabuli miserabiliter extincta.» - Blitzschläge haben zu allen Zeiten ihre Opfer gefordert und sind getreulich aufgezeichnet worden. Wenn gar noch beigefügt wird (Marbach 1824): «Es tonderte und blitzte 24 Stunden unaufhörlich», interessieren sich heute sogar Meteorologen für solche Hinweise. - Der Herkunft unserer Aufzeichungen - vorwiegend aus ländlichen Pfarreien entsprechend ist die Zahl jener Unfälle besonders groß, die irgendwie mit der Landwirtschaft zusammenhängen, so die der Leute, welche bei der Obsternte oder bei der Baumpflege abgestürzt waren. Da sticht einmal der ganz erhebliche Anteil der Frauen hervor, welche bei der Kirschenernte verunfallten; selbst alte Leute und Epileptiker ließ man noch die Bäume besteigen. So wird dem Namen einer über 60 Jahre alten Frau 1662 in Großwangen beigefügt: «Utrumque crus suum in duas partes confregit praeter grave vulnus conceptum in capite ... quatuor septimanas decumbens.» Im Herbst sind es dann vielfach Knaben, die herunterfallen, und zwar von Eichen. In Großwangen finden wir dafür 1664 eine Erklärung, die zugleich für die damalige Viehmast aufschlußreich ist. Ein $12 \mathrm{j}$. Knabe «decidit ex quercae arboris summitate, porcis siliquas decutiens». Tödliche Unfälle bei Arbeiten im Wald waren ebenfalls zahlreich und müssen immer einen tiefen Eindruck hinterlassen haben. In Pfeffikon widmet der Pfarrer 1797 zunächst dem 
Vorgehen beim Fällen eines Baumes viele Zeilen und fährt dann fort: «Inciput et occiput ex omnibus suturis usque ad os cribrosum nasale et tempora emota erant, cerebrum et cerebellum dispersa, contrita, in lucem prolapsa.» - Unfälle mit Fuhrwerken und Pferden sind die Vorläufer unserer Verkehrsunfälle. Die Wagen waren damals schmäler als heute und kippten auf den steinigen, unebenen Straßen um so leichter um. - Im Laufe des 17. Jahrhunderts wurden immer mehr Spieß- und Hellebardenträger in der Miliz auf Musketen umgerüstet. Jeder Hofbesitzer war verpflichtet, entweder selber mit einer Muskete am Trülltag zu erscheinen oder den Träger einer solchen Waffe auszurüsten.

Entsprechend fallen dann auch Schußverletzungen und gar Mörserexplosionen unter den Todesursachen auf, und wenn einmal die Umstände besonders eindrücklich sind und darum festgehalten werden, finden wir nicht einmal große Unterschiede gegenüber heute. Da steht in einem vornehmen Haushalt das Jagdgewehr geladen in der Stubenecke, der Hausvater setzt sich zum Nachmittagskaffee hin, ein Sohn richtet mutwillig die Waffe gegen ihn, drückt ab (Neudorf/Beromünster 1774). Heute noch werden anläßlich der Fronleichnamsprozession mit Mörsern religiöse Freudengefühle ausgedrückt. 1792 und 1810 geschah es schon mit üblen Folgen für die Bombardierer, ebenso 1752 und 1810 bei den Feiern zu Ehren neugewählter Äbte von Muri und St. Urban. - So unglaubhaft es klingen mag, eine Art von Unfällen, wie wir sie heute - unter ganz anderen kulturellen und emotionellen Voraussetzungen - miterleben, gab es in der guten alten Zeit auch schon. Über einen Vorfall, der sich in Einsiedeln anläßlich einer Wallfahrt ereignet hat, wird in Root 1731 berichtet: «In peregrinatione Einsiedlensi, dum prope januam S. Capellae devotionem suam perageret, a tumultuante concurrentis populi impetu ad proximioris columnae magnae angulum collisus subito expiravit.» Ähnlich mußte in Buttisholz 1812 ein 17j. Mädchen das Leben lassen «confluxu populi in ossorio obtrita occasione solemnitatis primitiarum missae».

Nach unseren eigenen Erfahrungen und Vorstellungen erwarten wir, in der alten Zeit auf eindrückliche Beispiele von Wundinfektion zu stoßen, kennen wir doch aus der einschlägigen Literatur das Schlagwort «pus bonum et laudabile». Am ehesten kommt unseren Kenntnissen noch die Formulierung «vulnere et febri» nahe (Neudorf 1769). In Hochdorf wird zwischen 1777 und 1830 häufig die Wendung «febris putrida» gebraucht. Wo aber sonst Infektion mit Eitererregern an irgendeiner Körperstelle zum Tod geführt hat, wird «apostema», allenfalls «gangraena» aufgezeichnet, so 
«fracto apostemate suffocata» 1665 und «gangraena in naso» 1632 , beide in Büron. Überhaupt fällt auf, wie oft bei «apostema» noch angegeben wird, der Verstorbene sei erstickt. War Lungen- allenfalls Tonsillarabszeß wirklich so häufig? In Sempach fällt der Gebrauch des Wortes «pus» auf, aus ein und derselben Feder um 1780 herum mit «pede putrescente», «inguine putrescente». Eine andere Wundkomplikation war damals gar nicht so selten, weil die üblichen Wundbehandlungsmittel gelegentlich und vor besonderen Bakterien versagten. Wohl wird sie in Sagen und Legenden erwähnt, und den Geistlichen muß sie aus Apg. 12,23 geläufig gewesen sein, wo es von Herodes (Agrippa I) heißt: «Percussit eum angelus Domini ... et consumptus a vermibus exspiravit.» In Römerswil wird 1696 «vermibus consumptus» verzeichnet, in Sempach über eine 80j. Frau 1776: «Exantlato acustissimo morbo et vix non viva vermibus depasta, crastino ob pessimum foetorem sepulta.» Volk und Geistliche machten ja keinen Unterschied zwischen Maden, Raupen und echten Wärmern; heute noch sind die Raupen der Kohlweißlinge in gutem Dialekt «Graswürmer», und die alten Schilderungen von «Würmern in Wunden» müßten zoologisch genauer von Fliegenmaden handeln.

\section{Verhältnis Arzt-Patient}

Selbst über Störungen im Verhältnis zwischen Arzt bzw. «operator» = Chirurg einerseits und dem Patienten oder dem Pfarrer anderseits finden sich recht eindrückliche Worte in den Totenbüchern. «Post longum incognoscibilem morbum» und «morbo ipsi medico ignoto» (Udligenswil 1792 und 1815) beleuchten diagnostische Schwierigkeiten ... begreiflich zu einer Zeit, in der es körperliche Untersuchungen so gut wie gar nicht gab. «Carne in saniem soluta ... histericum morbum fuisse dicunt medici insanabilem» läßt schon durchschimmern, daß der Pfarrer an den Fähigkeiten des Arztes gezweifelt hat (Sempach 1784). Ein anderes eindrückliches Beispiel haben wir oben im Abschnitt «Eklampsie» erwähnt. Noch deutlicher wird das mit den Worten unon provisa sacramentis repente obiit, iterum culpa medici dicentis morbum non esse periculosum» (Entlebuch 1770). An medizinische Berufsethik - würden wir heute sagen - rührt der Satz «per decem annos valetudinarius et medicorum ludibrium» bei einem 52j. Mann (Root 1787) und von derselben Hand schon 1777 «ab imperito animalium potius quam hominum chirurgo in collo operatus». 


\section{Krankenpflege}

War es schon üblich, daß Bettler in einem Stall übernachten mußten und dort Kinder zur Welt brachten oder starben, so ist es nicht verwunderlich, daß es auch für unruhige und unsaubere Geisteskranke keinen anderen Aufenthaltsort gab. Eindrücklich ist, was uns 1720 aus Römerswil über einen solchen Fall erhalten geblieben ist: «Per plurimos annos catena ligatus propter continuum delirium.» Das ist nicht der einzige Fall, der uns so grausame Umstände aufzeigt.

Vom Pfarrer besonders hervorgehoben wurde aber auch, wenn sich Frauen bei der Pflege von Kranken besonders hervorgetan und sich dabei selber den Tod geholt hatten. "Quae fratri nuper defuncto in morbo inservierat» und «correpta morbo quo aegrotae A.M.A. ministraverat» (Romoos 1761 und 1757). «Per plures annos misere lecto affixus omnibus membris contractus, in suo miserabili statu a communitate in domo sororis suae sustentatus» zeigt heute noch das Schicksal des zum Pflegefall gewordenen Polyarthritikers (Root 1791). Noch deutlicher werden die schwierigen Umstände damaliger Krankenpflege beleuchtet von den Worten: «... ob spurcitiam et inde ortum foetorem refugiente humana ope» wurde eine $50 \mathrm{j}$. Patientin doch noch von einer mitleidigen Frau zu Tode gepflegt.

\section{Quellen}

Sterbebücher luzernischer Pfarreien bis 1875 auf Mikrofilmen im Staatsarchiv Luzern nach dort aufliegendem Verzeichnis

\section{Literatur}

1 Historisch-Biographisches Lexikon der Schweiz, Neuenburg 1924 II, 338

2 Schürmann Joseph, Mitteilungen der Naturforschenden Gesellschaft Luzern XXV, 179 (1977)

3 Eschle Alfons, Ärzte und Arztwesen im Entlebuch, Schüpfheim 1937 


\section{Zusammenfassung}

Sterbebücher aus den katholischen Luzerner Pfarreien, ca. 1600-1875, enthalten vielfache, aber unsystematische Angaben über Ursachen und Umstände von Unfällen, über Einzelheiten aus der Geburtshilfe, heute noch übliche Diagnosen neben Einzelheiten, welche nach den Unständen erlauben, nachträglich Diagnosen zu stellen wie Ileus nach Appendicitis, Prostatahypertrophie, Eklampsie, Placenta praevia, Wehenschwäche post partum usw. und sogar Hinweise auf Trübung des Verhältnisses zwischen Arzt und Patient bzw. zwischen Arzt und Pfarrer.

\section{Summary}

The bills of mortality or obituaries («Sterbebücher») of Catholic Lucerne hold many but not systematic details concerning the circumstances and causes of death. We read about accidents, about obstetric incidents, ileus post appendicitem, adenoma of the prostate, eclampsia, placenta praevia and haemorrhagia post partum. There are furthermore even some hints about the relation between patient and doctor or about the rector and the doctor.

Dr. med. Joseph Schürmann-Roth

Seefeldstraße 6

CH-6006 Luzern 